\title{
Endoplasmic reticulum stress and autophagy dysregu- lation in alcoholic and non-alcoholic liver diseases
}

\author{
Yun Seok Kim¹ and Sang Geon Kim¹,2 \\ 'College of Pharmacy, Seoul National University, Seoul; ${ }^{2}$ College of Pharmacy, Dongguk University, Goyang, Korea
}

Alcoholic and non-alcoholic liver diseases begin from an imbalance in lipid metabolism in hepatocytes as the earliest response. Both liver diseases share common disease features and stages (i.e., steatosis, hepatitis, cirrhosis, and hepatocellular carcinoma). However, the two diseases have differential pathogenesis and clinical symptoms. Studies have elucidated the molecular basis underlying similarities and differences in the pathogenesis of the diseases; the factors contributing to the progression of liver diseases include depletion of sulfhydryl pools, enhanced levels of reactive oxygen and nitrogen intermediates, increased sensitivity of hepatocytes to toxic cytokines, mitochondrial dysfunction, and insulin resistance. Endoplasmic reticulum (ER) stress, which is caused by the accumulation of misfolded proteins and calcium depletion, contributes to the pathogenesis, often causing catastrophic cell death. Several studies have demonstrated a mechanism by which ER stress triggers liver disease progression. Autophagy is an evolutionarily conserved process that regulates organelle turnover and cellular energy balance through decomposing damaged organelles including mitochondria, misfolded proteins, and lipid droplets. Autophagy dysregulation also exacerbates liver diseases. Thus, autophagy-related molecules can be potential therapeutic targets for liver diseases. Since ER stress and autophagy are closely linked to each other, an understanding of the molecules, gene clusters, and networks engaged in these processes would be of help to find new remedies for alcoholic and non-alcoholic liver diseases. In this review, we summarize the recent findings and perspectives in the context of the molecular pathogenesis of the liver diseases. (Clin Mol Hepatol 2020;26:715-727)

Keywords: Non-alcoholic steatohepatitis; Non-alcoholic fatty liver disease; Mitochondria; Endoplasmic reticulum stress; Autophagy

\section{INTRODUCTION}

Excessive and chronic alcohol consumption may lead to the progression of alcoholic liver disease (ALD).' ALD is caused by chronic alcohol consumption (>20 g/day for women and $>30 \mathrm{~g} /$ day for men). ${ }^{2,3}$ Moreover, repeated alcohol drinking may promote progression of simple steatosis to steatohepatitis and/or cirrhosis. ${ }^{4}$
Another most common liver disease is non-alcoholic fatty liver disease (NAFLD), which is considered as the hepatic manifestation of the metabolic syndrome including hypertension, type 2 diabetes, insulin-resistance, obesity, dyslipidemia, and distinct hepatic histological features. ${ }^{5}$ Both ALD and NAFLD share general histopathological spectrum from simple steatosis to steatohepatitis and fibrosis, which may progress to more severe diseases (i.e., cir-

\author{
Abbreviations: \\ $A L D$, alcoholic liver disease; $E R$, endoplasmic reticulum; FFAs, free fatty acids; \\ Ga12, G protein subunit alpha 12; HSCs, hepatic stellate cells; LXRa, liver X \\ receptor $a$; miR, microRNA; NAD, nicotinamide adenine dinucleotide; $N A D H$, \\ nicotinamide adenine dinucleotide hydride; NAFLD, non-alcoholic fatty liver \\ disease; NASH, non-alcoholic steatohepatitis; ROS, reactive oxygen species
}

\author{
Corresponding author : Sang Geon Kim \\ College of Pharmacy, Dongguk University, 32 Dongguk-ro, Ilsandong-gu, \\ Goyang 10326, Korea \\ Tel: +82-31-961-5218, Fax: +82-31-961-5206 \\ E-mail: sgkim@dongguk.edu \\ https://orcid.org/0000-0001-5266-1722
}


rhosis and hepatocellular carcinoma) (Fig. 1). ${ }^{4}$ However, the two diseases differ from each other in a variety of properties, ranging from the molecular mechanisms of disease exacerbation to differences in clinical features. In particular, infiltration of inflammatory cells occurs to a greater degree in ALD than in NAFLD. In contrast,

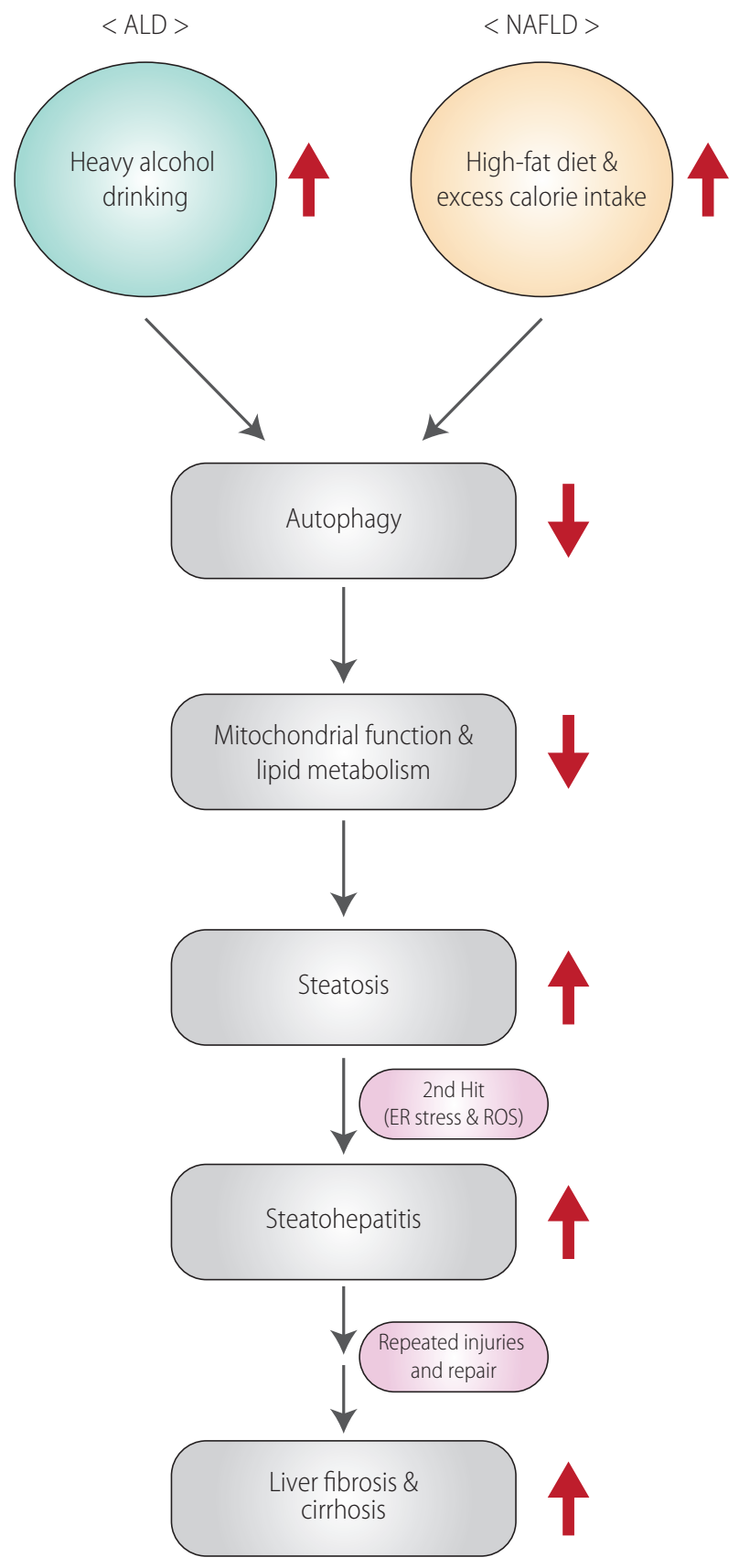

Figure 1. General pathological processes for ALD and NAFLD. ALD, alcoholic liver disease; NAFLD, non-alcoholic fatty liver disease; ER, endoplasmic reticulum; $R O S$, reactive oxygen species. fat degeneration in hepatocytes is more pronounced in NAFLD than in ALD. ${ }^{6}$ Despite the ongoing study of the pathology, causes, and risk factors for the diseases, we do not yet have an appropriate treatment regimen. Here, we aim to motivate intensive research on the diseases by reviewing the current understanding of the causes of ALD and NAFLD, and the trends in potential therapeutic approaches.

\section{GENERAL MOLECULAR PATHOGENESIS \\ Definition of endoplasmic reticulum (ER) stress}

ER is a structure of a membrane component that extends from the nuclear membrane and has two types: a ribosome attached rough ER and a ribosome-free smooth ER. Approximately onethird of the protein in the cell is translated from the messenger RNA to protein, which becomes an active protein structure through processes such as folding, assembly, glycation, and disulfide bond. ' Smooth ER is the site for the synthesis of lipids and sterols, and functions as a calcium reservoir to regulate the cellular calcium concentrations. ${ }^{8,9}$ However, if either immature protein flows into the ER above the capacity, or calcium is depleted in the $E R$, the function of $E R$ is impaired. This condition is defined as $E R$ stress. ${ }^{9.11}$ When ER stress occurs, cells have a defense mechanism to survive, which is called the ER stress response. ${ }^{8}$ Thus, ER stress is a cellular stress, in which the levels of synthetic proteins exceed the capacity of unfolded protein responses. Finally, when the ER stress becomes so severe that it cannot be overcome and the ER cannot recover its function, then cell death pathway is activated with inflammatory responses. ${ }^{9,12}$

\section{Cross-links between ER and mitochondria}

In several cell and animal models, we found ER stress as a key stimulus of fatty liver transition to non-alcoholic steatohepatitis (NASH). So, ER stress is claimed as a secondary hit to provoke hepatocyte injury and inflammation, leading to liver fibrosis in a chronic situation (Fig. 1). Dysregulation of unfolded protein responses enhances ER stress and consequently may promote catastrophic cell death. ${ }^{13}$ Additionally, ER stress, in association with reactive oxygen species (ROS) production, triggers the subsequent activation of cell death pathway due to imbalance of redox homeostasis. ${ }^{14,15}$ Cytochrome P450 2E1, a high affinity enzyme for ethanol metabolism, is located in the membrane of ER, and thus 
Yun Seok Kim, et al.

ER stress and autophagy in liver diseases

the induction of cytochrome P450 2E1 would be also associated with ER stress in hepatocytes inflicted by sustained ethanol consumption. ${ }^{16}$ This idea would be also supported by impairment of free fatty acids (FFAs) oxidation in mitochondria because mitochondria and ER physiologically work together through mitochondria-associated ER membrane. ${ }^{17}$

The lipids are mainly metabolized in the liver, and lipid metabolism in the liver maintains homeostasis by balancing or storing fat for energy. ${ }^{18}$ In particular, lipid absorption, esterification, oxidation and secretion of fatty acids are performed in liver cells. ${ }^{19}$ The supply of FFAs to the liver contributes to the pathogenesis of hepatic steatosis; triglycerides and cholesterol are stored in the form of lipid droplets to protect cells from exposure to excessive amounts of FFAs that can damage signal pathways and metabolic homeostasis. ${ }^{20}$ Impairment of oxidative metabolism of FFAs is also coupled to fat accumulation because FFAs are used as fuel for mitochondrial oxidation.

Compared to normal individuals, the incidence of ALD increases in patients with fatty liver by 2-3 times. ${ }^{21}$ Thus, obesity is considered as an independent risk factor for alcoholic steatohepatitis since diet pattern and dietary fat content contribute to the progression of ALD. Mitochondrial dysfunction occurs not only by peroxidation of unsaturated fats of mitochondrial phospholipids, but by the attack of oxygen free radicals generated through ethanol metabolism. Hence, excessive alcohol consumption causes impairment of mitochondrial function. ${ }^{22}$ In NAFLD patients, dysregulation of fatty acid metabolism causes hepatic steatosis and hyperlipidemia in a large patient population. In addition, NAFLD as a result of obesity and diabetes facilitates inflammatory cytokines production. ${ }^{23}$ So, metabolic profile in hepatocytes greatly alters because of fuel source and consumption rates change. Together, metabolic disturbances caused by fat along with alcohol consumption stimulate worsening of alcoholic steatohepatitis, which may lead to more severe conditions.

\section{MITOCHONDRIAL TARGETS}

Peroxisome proliferator-activated receptor alpha regulates the expression of lipid metabolism-related genes (e.g., Cyp4a1, Acbp, and $A(s)) .{ }^{24}$ In our preliminary study, we found that mitochondrial activity and oxidative energy metabolism are controlled by core molecules including sirtuin 1 , peroxisome proliferator-activated receptor- $y$ coactivator 1-alpha and peroxisome proliferator-activated receptor alpha (i.e., a transcription complex required for mi- tochondrial biogenesis). ${ }^{25,26}$ In this study, we have shown that an activated form of $\mathrm{G}$ protein subunit alpha 12 (Ga12) stabilizes sirtuin 1 via ubiquitin specific peptidase 22 induction, which depends on hypoxia-inducible factor 1-alpha. The research results showed that Ga12 regulates sirtuin 1-dependent lipid oxidation in mitochondria through hypoxia-inducible factor 1-alpha-mediated ubiquitin specific peptidase 22 induction, identifying Ga12 as a linker between cell surface signaling and mitochondrial lipid oxidation (Fig. 2).

Liver fibrosis is manifested by repeated wound healing processes, such as an increase in matrix protein and a decrease in matrix remodeling: liver fibrosis is characterized by decreased matrix remodeling and increased production of matrix proteins, and may lead to end-stage cirrhosis. ${ }^{27}$ Liver fibrosis is defined by concerted actions of non-parenchymal cells of the liver, particularly macrophages (including Kupffer cells), hepatic stellate cells (HSCs), and endothelial cells. ${ }^{28}$ Accumulation of aberrant extracellular matrix is induced by a diverse population of myofibroblasts, among which HSCs play a major role. ${ }^{29}$

\section{AUTOPHAGY TARGETS}

\section{Definition of autophagy}

Studies have reported the correlation between hepatic diseases and autophagy. Autophagy may inhibit the progression of steatosis and fatty hepatitis by preventing hepatocyte injury. 30,31

The liver is the major detoxifying and metabolic organ. Autophagy, a "recycling mechanism" in hepatocytes, is evolved to salvage key metabolites and to provide energy for sustaining anabolism. ${ }^{32}$ Thus, autophagy is a crucial regulator of cellular homeostasis; ${ }^{32-34}$ autophagy targets damaged cellular constituents, such as denatured proteins, accumulated fat, and mitochondria that have lost their function to lysosomes for degradation. ${ }^{35,36}$ Amino acids, insulin, and mammalian target of rapamycin signaling pathways inhibit the autophagic pathway. ${ }^{36}$ Additionally, fasting regulates metabolic pathways, such as gluconeogenesis, ketone body formation, and $\beta$-oxidation. Fatty acids are mainly generated by selective autophagy of lipid droplets (lipophagy), while amino acids are generated by proteolysis through autophagy and are used for gluconeogenesis. ${ }^{33,37}$ Therefore, autophagy plays a decisive role in regulating liver physiology and balancing liver metabolism. ${ }^{38}$ 


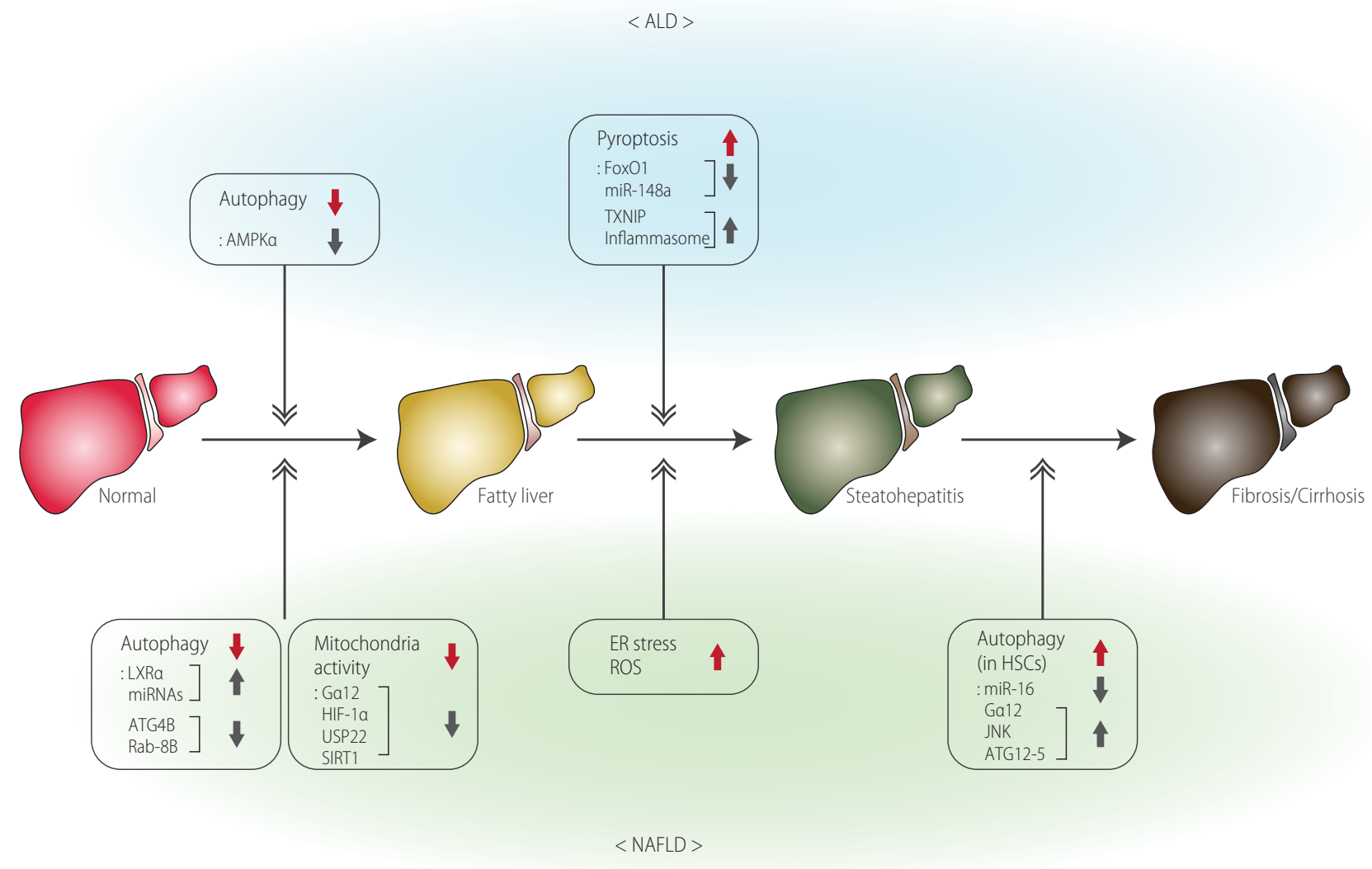

Figure 2. Functional molecular biomarkers for the processes of ALD and NAFLD. ALD, alcoholic liver disease; AMPKa, AMP-activated protein kinase alpha; miR, microRNA; TXNIP, thioredoxin interacting protein; LXRa, liver X receptor a; ATG4B, autophagy related 4B cysteine peptidase; Ga12, G protein subunit alpha 12; HIF-1a, hypoxia-inducible factor 1-alpha; USP22, ubiquitin specific peptidase 22; SIRT1, sirtuin 1; ER, endoplasmic reticulum; ROS, reactive oxygen species; HSCs, hepatic stellate cells; JNK, c-Jun N-terminal kinase; ATG12-5, autophagy related 12-5 conjugate; NAFLD, non-alcoholic fatty liver disease.

\section{Autophagic molecules associated with liver steatosis}

Chronic alcohol intake or excessive energy intake causes damage to the regulation of autophagy. When it persists, the homeostatic balance breaks and metabolic diseases develop. ${ }^{39,40} \mathrm{Al}-$ though several studies have demonstrated the correlation between autophagy and metabolic liver disease, the role of autophagy in metabolic liver disease has not been completely elucidated. Moreover, the understanding of the regulatory molecules by which the disease progression is determined is still needed.

The role of autophagy in acute and chronic ALD is still complex and controversial. It is generally known that binge drinking enhances autophagy of hepatocytes, which limits hepatic cell damage, death and fat accumulation by selectively removing excess lipid droplets and damaged mitochondria. ${ }^{41}$ In contrast, chronic alcohol intake has been reported to decrease lysosome function and increase ubiquitinated aggregates accumulation. ${ }^{42}$ Thus, the duration of alcohol exposure affects autophagy. ${ }^{43} 5^{\prime}$ AMP-activated protein kinase, an enzyme that plays a role in the metabolism of fatty acids (e.g., acetyl-CoA carboxylase), is a key player of autophagy. Ethanol consumption causes inhibition of $5^{\prime}$ AMP-activated protein kinase, suggestive of the role of autophagy in ALD. One of the studies claims a mechanism for suppressing autophagy during chronic alcohol intake in association with decrease of $5^{\prime}$ AMP-activated protein kinase activity, which may increase fat production through decrease of $\beta$-oxidation. ${ }^{44}$ This information can be employed to understand the pathogenesis of ALD.

Although the evaluation of autophagy in animal and human samples is somewhat controversial, ${ }^{45}$ it is generally agreed that autophagy is reduced in NAFLD. Hepatocellular steatosis is aggravated in mice deficient in autophagy-related genes. ${ }^{46,47}$ Inhibition of hepatocyte-specific run domain beclin-1-interacting and cysteine-rich domain-containing protein (beclin-1 interacting negative regulator for autophagosome fusion), overexpression of adenovi- 
Yun Seok Kim, et al.

ER stress and autophagy in liver diseases

ral-induced autophagy-related $7,{ }^{47,48}$ or treatment of an autophagy activator such as rapamycin can relieve ER stress and hepatic steatosis. The opposite effect was seen with the inhibitor. ${ }^{49}$ Moreover, autophagy participates in the basic conversion process of lipids by decomposing lipid droplets under physiological conditions. Recently, we demonstrated that liver $X$ receptor a (LXRa) functions as a transcriptional regulator of microRNAs (miRs). ${ }^{50}$ In this study, gene set enrichment analysis was used to discover the phenomenon that autophagy can be inhibited by $L X R a$, and focusing on this, we proved the regulatory mechanism of autophagy using animal model. As a mechanistic basis, it was noted that LXRa inhibits autophagy-related 4B cysteine peptidase and rasrelated protein $R a b-8 B$, and this effect is due to the transcriptional induction of let-7a and miR-34a by LXRa. In addition, the same mechanism works in human samples and genetic obesity-derived animal $(o b / o b, d b / d b)$ samples. Furthermore, LXRa and the above identified molecules all inhibit lipid degradation and mitochondrial function, supportive of a link between autophagy and NAFLD when LXRa is activated. The outcomes may provide key information for target discovery and potential strategy to treat NASH (Fig. 2).

\section{Autophagic molecules associated with liver fibrosis}

Autophagy is reported to be involved in the progression of liver fibrosis. ${ }^{51}$ Activation of HSCs is a major phenomenon in liver fibrosis because these cells differentiate into myofibroblasts and produce a major extracellular matrix in the liver. ${ }^{52} \mathrm{HSCS}$ of the quiescent phenotype store vitamin $A$ in lipids and are activated when vitamin $A$ is decomposed by various stimuli. Thus, HSCs can be activated by promoting autophagy. Previously, we have shown that Ga12 activation increases autophagy in $\mathrm{HSCS}$, accompanying c-Jun N-terminal kinase-dependent autophagy-related 12-5 conjugation. ${ }^{53}$ In the study, miR-16 directly inhibits de novo synthesis of Ga12 and thereby modulations of miR-16 alter autophagy (Fig. 2). Consistently, patients with severe fibrosis exhibited downregulated levels of miR-16. ${ }^{53}$ The activation of HSCs by apoptotic hepatocytes or by transforming growth factor- $\beta$ produced from activated Kupffer cells also contributes to fibrosis. ${ }^{54}$ In this process, sustained ER stress, cytokines, saturated FFAs, and adipokines could be also involved. ${ }^{54}$

\section{Potential targeting of autophagy}

Currently, there are no specific treatments for non-alcohol/alcohol-related liver disease, and the main treatment is dietary control or alcohol withdrawal. Recent studies have demonstrated the importance of autophagy in metabolic diseases and suggested new treatment strategies. Preclinical studies have reported that enhancing autophagy using carbamazepine or rapamycin decreases fat accumulation and liver damage in obese and chronic ethanolfed mice. ${ }^{49}$ Although there is a Janus face of autophagy in chronic liver disease, autophagy is primarily a hepatoprotective mechanism; autophagy is protective against the early stages of hepatocellular carcinoma and promotes liver regeneration. ${ }^{55}$ Conversely, autophagy may be deleterious during the late phase of hepatocellular carcinoma in addition to activation of HSCs. ${ }^{51,56}$ Thus, the cell-specific and pathology-specific regulation of autophagy should be considered for devising strategies for liver disease treatment.

\section{CHANGES IN LIPID PROFILES}

Alcohol-induced hepatic dysfunction or injury is closely related to abnormal lipid profiling. ${ }^{57,58}$ Cumulated evidence shows that liver steatosis is linked to insulin resistance and the consequent hyperinsulinemia. In addition to steatosis, chronic alcohol consumption induces insulin resistance in the liver as indicated by the suppression of insulin-responsive genes. ${ }^{59,60}$ Moreover, heavy alcohol consumption may lead to impairment of mitochondrial membrane depolarization; dysregulation of cellular redox state results at least in part from changes in the nicotinamide adenine dinucleotide $\left(N A D^{+}\right) /$nicotinamide adenine dinucleotide hydride (NADH) ratio. ${ }^{61,62}$ Alcohol disrupts the sirtuin 1 signaling pathway, which is involved in the regulation of mitochondrial biogenesis and oxygen consumption, and consequently promotes hepatic steatosis, injury, and inflammation. ${ }^{63}$ In particular, mitochondrial dysfunction contributes to this change to a large extent, in which replacement of old non-functional mitochondria is disturbed due to autophagy inhibition as well as impaired proteasome function. ${ }^{64,65}$ Electron microscopy has revealed the presence of megamitochondria in the liver of patients with ALD. ${ }^{66,67}$

Lipotoxicity not only causes inflammation, ER stress, and ROS, but also affects the biological function of organelles, the most important of which is the mitochondria. ${ }^{68}$ Increasing autophagy removes damaged mitochondria in a non-alcoholic fatty liver animal model, improving fatty liver. ${ }^{69}$ Conversely, when autophagy is inhibited, severe mitochondrial damage and hepatic steatosis occur. ${ }^{70}$ Additionally, suppression of autophagy through drug or gene regulation can reduce the number of triglycerides and lipid 


\section{CLINICAL and MOLECULAR}

Volume_26 Number_4 October 2020

Table 1. Ongoing clinical trials or candidate targets of pharmacotherapies for the treatment of ALD or NAFLD

\begin{tabular}{|c|c|c|c|c|}
\hline Pathology & Target & Agent & Stage & Reference \\
\hline \multirow[t]{20}{*}{ ALD } & Hepatic regeneration & IL-22 (F-652) & Phase 1 & 100 \\
\hline & IgG antibody to LPS & Bovine colostrum (IMM-124E) & Phase 2 & 101 \\
\hline & Probiotic, restores gut microbiome & Lactobacillus rhamnosus GG & Phase 2 & 102 \\
\hline & Antagonist to IL-1 receptor & Anakinra & Phase 2 & 103 \\
\hline & FXR agonism & Obeticholic acid (INT-747) & Phase 2 & 104 \\
\hline & Antibiotic amoxicillin plus clavulanic acid & Augmentin & Phase 3 & 105 \\
\hline & Antioxidant and promotes abstinence & Metadoxine & Phase 4 & 106 \\
\hline & Increase neutrophils, hepatic regeneration & G-CSF (filgrastim) & Phase 4 & 107 \\
\hline & Glutamate & - & Target discovery stage & 108 \\
\hline & HMGB1 & - & Target discovery stage & 109 \\
\hline & $\mathrm{ALDH} 2$ & - & Target discovery stage & 110 \\
\hline & Toll-like receptor 3 & - & Target discovery stage & 111 \\
\hline & C3aR, C5aR1 & - & Target discovery stage & 112 \\
\hline & Fructose, cytochrome P450-2E1 & - & Target discovery stage & 113 \\
\hline & HIF-1a & - & Target discovery stage & 114 \\
\hline & Lactobacillus GG & - & Target discovery stage & 115 \\
\hline & Bile acid, FXR, FGF15 & - & Target discovery stage & 116 \\
\hline & FGF19 & - & Target discovery stage & 117 \\
\hline & Fox01, miR-148a, TXNIP & - & Target discovery stage & 91 \\
\hline & REG3 lectins & - & Target discovery stage & 118 \\
\hline \multirow[t]{21}{*}{ NAFLD } & Thyroid hormone receptor beta agonist & VK2809 & Phase 2 & 119 \\
\hline & PanPPAR agonist & Lanafibranor & Phase 2 & 120 \\
\hline & FXR agonist and SGLT1/2 inhibitor & Tropifexor and licoglifozin & Phase 2 & 121 \\
\hline & Engineered version of human hormone FGF19 & Aldafermin & Phase 2 & 122 \\
\hline & FXR agonist & EDP-305 & Phase 2 & 123 \\
\hline & ASBT inhibitor & Volixibat & Phase 2 & 124 \\
\hline & Recombinant FGF21 & BMS-986036 & Phase 2 & 125 \\
\hline & Pan-caspase inhibitor & Emricasan & Phase 2 & 126 \\
\hline & Galectin 3 inhibitor & GR-MD-02 & Phase 2 & 127 \\
\hline & CCR2/CCR5 receptor inhibitor & Cenicriviroc (AURORA) & Phase 3 & 128 \\
\hline & SCD1 modulator & Aramchol & Phase 3 & 129 \\
\hline & Thyroid hormone receptor beta agonist & Resmetirom & Phase 3 & 130 \\
\hline & PPARa/ठ ligand & Elafibranor (RESOLVE-IT) & Phase 3 & 131 \\
\hline & FXR ligand & Obeticholic acid (REGENERATE) & Phase 3 & 132 \\
\hline & FXR agonist & Obeticholic acid (REVERSE) & Phase 3 & 132 \\
\hline & ASK1 inhibitor & Selonsertib & Phase 3 & 133 \\
\hline & SGLT2 inhibitor & Gliflozin & Pilot & 134 \\
\hline & LXRa, let-7a, miR-34a, ATG4B, Rab-8B & - & Target discovery stage & 50 \\
\hline & STAT-1, STAT-3 & - & Target discovery stage & 135 \\
\hline & Fructokinase & - & Target discovery stage & 136 \\
\hline & Ga13, ITIH1 & - & Target discovery stage & 137 \\
\hline
\end{tabular}


Table 1. Continued

\begin{tabular}{llllll}
\hline Pathology & Target & & Agent & Stage & Reference \\
\hline & & - & Target discovery stage & 138 \\
TAZ & - & Target discovery stage & 139 \\
TNFAIP3 & - & Target discovery stage & 140 \\
Glutaminase 1 & - & Target discovery stage & 26 \\
USP22, Ga12, SIRT1 & - & Target discovery stage & 141 \\
OTULIN & & &
\end{tabular}

ALD, alcoholic liver disease; NAFLD, non-alcoholic fatty liver disease; IL, interleukin; IgG, immunoglobulin G; LPS, lipopolysaccharide; FXR, farnesoid X receptor; G-CSF, granulocyte-colony stimulating factor; HMGB1, high mobility group box-1; ALDH2, aldehyde dehydrogenase 2; HIF, hypoxia-inducible factor; FGF, fibroblast growth factor; miR, microRNA; TXNIP, thioredoxin interacting protein; REG3, regenerating islet-derived protein 3; PanPPAR, pan-peroxisome proliferator-activated receptor; SGLT, sodium-glucose co-transporter; ASBT, apical sodium-dependent bile acid transporter; $C C R, C-C$ chemokine receptor; SCD, stearoyl-COA desaturase; PPAR, peroxisome proliferator-activated receptor; ASK1, apoptosis signal-regulating kinase 1; LXRa, liver $X$ receptor a; ATG4B, autophagy related 4B cysteine peptidase; STAT, signal transducer and activator of transcription; Ga13, G protein subunit alpha 13; ITIH, inter-alpha-trypsin inhibitor heavy chain H1; TAZ, tafazzin; TNFAIP3, tumor necrosis factor alpha induced protein 3; USP, ubiquitin specific peptidase 22; SIRT, sirtuin 1; OTULIN, OTU domain-containing deubiquitinase with linear linkage specificity.

particles in hepatocytes. ${ }^{71}$ As a result, a decrease in FFAs supply from lipid particles reduces mitochondrial $\beta$-oxidation, and the consequential fat accumulation in turn increases mitochondrial dysfunction. $^{72}$

Therefore, the interactions among mitochondrial activity, biogenesis, and autophagy contribute to metabolic dysregulation of hepatocytes in alcoholic and non-alcoholic liver diseases.

\section{ALD AND DRUG CANDIDATES}

Alcoholic liver steatosis is considered as a benign condition and is thus reversible to normal state under the condition of abstinence. ${ }^{73,74}$ One of the early hallmarks of alcohol-induced liver damage caused by alcohol is the accumulation of lipid droplets in hepatocytes. ${ }^{75}$ Clinically, most patients experience no symptoms for alcohol-induced fatty liver. However, patients with alcoholic steatohepatitis or fibrosis may present symptoms such as nausea and vomiting, which are caused by dysregulated cytokines production. ${ }^{76-79}$ In a small proportion of chronic and heavy alcohol drinkers, ALD may lead to the development of liver cirrhosis (Fig. 1). ${ }^{80-84}$

Electrons generated during alcohol metabolism promote production of reactive intermediates, including ROS and reactive nitrogen species, which are catalyzed by enzymes such as alcohol dehydrogenase and cytochrome P450 2E1. ${ }^{85,86}$ ROS and reactive nitrogen species oxidize lipids to generate lipid peroxidation end products such as isoprostane and malondialdehyde. ${ }^{87-89}$ Mitochondria is another organelle, from which ROS is greatly generated through oxidative fuel consumption. ${ }^{90}$ Since an excess amount of ROS production causes depletion of reduced sulfhydryl pools (e.g., glutathione) in hepatocytes, hepatocytes may be sensitized by the stresses of toxic cytokines (e.g., tumor necrosis factor- $\beta$ ) and thus are more susceptible to injurious challenges. Previously, we had demonstrated that a spectrum of liver injury, including pyroptotic hepatocyte death, occurs in an alcohol abuse animal model; alcohol induces pyroptosis in hepatocytes by promoting 'thioredoxin interacting protein and NOD-, LRR- and pyrin domain-containing protein $3^{\prime}$ inflammation through decreases of miR-148a and forkhead box protein 01. ${ }^{91}$ Attention was also paid to methionine and S-adenosylmethionine in the field of metabolic mechanism studies, the levels of which may be changed by alcohol consumption as part of the evidence of changes in cellular glutathione pool..$^{92}$

As mentioned above, various mechanisms are involved in ALD, and based on these findings, clinical treatment with new and emerging molecular targets for ALD treatment is ongoing (Table 1).

\section{NAFLD AND DRUG CANDIDATES}

Globally, NAFLD is the most common liver disease that encompasses diseases from inflamed steatosis to NASH, cirrhosis, and hepatocellular carcinoma. ${ }^{93}$ In Asian and American populations, the prevalence of NAFLD is approximately $30 \%$ and the disease is often accompanied by type 2 diabetes and obesity. ${ }^{94}$ Accurate diagnosis of NASH is essential because inflammation and/or fibrosis may determine the prognosis of NASH. Additionally, advanced conditions such as cirrhosis may require liver transplantation accordingly. ${ }^{95}$ Currently, there are increased efforts to develop thera- 
peutic strategies for NASH, which have no established treatment.

Previous studies have reported that multiple hits triggered NAFLD, which was recognized as a potential therapeutic goal. Several modulators of these targets and related pathways have been analyzed in clinical trials (phase II) or are currently in ongoing clinical trials (Table 1). ${ }^{93}$ The candidate drugs inhibit de novo lipogenesis or increase lipid export from the liver. ${ }^{94}$ Additionally, liver or systemic insulin resistance is improved by diverse drugs or gastrointestinal hormones such as peroxisome proliferator-activated receptor $\gamma$ agonists. Accumulation of excess lipids may facilitate simple steatosis progression to inflamed steatosis. This step usually provides effective targeting because inflammation generally precedes fibrosis. Currently, the inhibitors of apoptosis signalregulating kinase $1, \mathrm{C}-\mathrm{C}$ chemokine receptor types $2 / 5$, and chemokine receptors of $\mathrm{C}-\mathrm{C}$ motif chemokine 5 and $\mathrm{C}-\mathrm{C}$ motif chemokine 2 are under phase III clinical trials. ${ }^{96}$ Several farnesoid $X$ receptor agonists reduce gluconeogenesis and liver fat production, inhibit bile acid synthesis, improve peripheral insulin sensitivity, and thereby have a profound effect on various pathways for development of NAFLD. ${ }^{97,98}$ Several farnesoid $X$ receptor agonists have been tested in clinical trials (Table 1). ${ }^{99}$ Combination therapy may be an effective therapeutic strategy for NAFLD as it has a complex pathophysiology.

\section{CONCLUSION}

In this review, we attempted to summarize the molecular mechanisms underlying ALD and NAFLD. Both diseases are associated with unhealthy lifestyle habits, including increased consumption of alcohol and unhealthy diet. Extraordinary progress has been made in the understanding of the pathology of fatty liver disease so far, suggesting that several parallel hits are required to conquer the disease. Understanding the molecular mechanisms underlying ALD and NAFLD is important for medical professionals, especially physicians, general practitioners, hepatologists, and diabetologists. Because fatty liver disease has many clinical features, doctors should consider the patients in a multifaceted way. Identifying the various molecular mechanisms and finding therapeutic functions are key clinical prerequisites. In the future, the awareness of the disease progression and key targets would enable proper diagnosis, effective management, and treatment of comorbid diseases.

\section{Authors' contribution}

YSK and SGK contributed to the literature review, and manuscript preparation.

\section{Acknowledgements}

This work was supported by the National Research Foundation of Korea (NRF) grant funded by the Korea government (MSIP) (NRF-2018R1A2A1A05078694) and in part by Dongguk University fund.

\section{Conflicts of Interest}

The authors have no conflicts to disclose.

\section{REFERENCES}

1. Ramaiah S, Rivera C, Arteel G. Early-phase alcoholic liver disease: an update on animal models, pathology, and pathogenesis. Int J Toxicol 2004;23:217-231.

2. Mathurin $P$, Bataller R. Trends in the management and burden of alcoholic liver disease. J Hepatol 2015;62(1 Suppl):S38-S46.

3. Zakhari S, Li TK. Determinants of alcohol use and abuse: impact of quantity and frequency patterns on liver disease. Hepatology 2007;46:2032-2039.

4. Gual P, Gilgenkrantz H, Lotersztajn S. Autophagy in chronic liver diseases: the two faces of Janus. Am J Physiol Cell Physiol 2017;312:C263-C273.

5. Younossi Z, Tacke F, Arrese M, Chander Sharma B, Mostafa I, Bu-

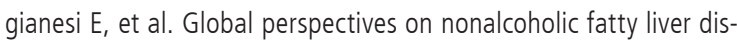
ease and nonalcoholic steatohepatitis. Hepatology 2019;69:26722682.

6. Tannapfel A, Denk H, Dienes HP, Langner C, Schirmacher P, Trauner $M$, et al. Histopathological diagnosis of non-alcoholic and alcoholic fatty liver disease. Virchows Arch 2011;458:511-523.

7. Kaufman RJ. Stress signaling from the lumen of the endoplasmic reticulum: coordination of gene transcriptional and translational controls. Genes Dev 1999;13:1211-1233.

8. Schröder M, Kaufman RJ. The mammalian unfolded protein response. Annu Rev Biochem 2005;74:739-789.

9. Oyadomari S, Araki E, Mori M. Endoplasmic reticulum stress-mediated apoptosis in pancreatic beta-cells. Apoptosis 2002;7:335-345.

10. Mori K. Tripartite management of unfolded proteins in the endoplasmic reticulum. Cell 2000;101:451-454.

11. Kaufman RJ, Scheuner D, Schröder $M$, Shen $X$, Lee $K$, Liu CY, et al. The unfolded protein response in nutrient sensing and differentiation. Nat Rev Mol Cell Biol 2002;3:411-421.

12. Araki E, Oyadomari S, Mori M. Impact of endoplasmic reticulum 
stress pathway on pancreatic beta-cells and diabetes mellitus. Exp Biol Med (Maywood) 2003;228:1213-1217.

13. Xu C, Bailly-Maitre B, Reed JC. Endoplasmic reticulum stress: cell life and death decisions. J Clin Invest 2005;115:2656-2664.

14. Lieber CS. Hepatic, metabolic and toxic effects of ethanol: 1991 update. Alcohol Clin Exp Res 1991;15:573-592.

15. Grunnet N, Kondrup J. The effect of ethanol on the beta-oxidation of fatty acids. Alcohol Clin Exp Res 1986;10(6 Suppl):64S-68S.

16. Cho YE, Mezey E, Hardwick JP, Salem N Jr, Clemens DL, Song BJ. Increased ethanol-inducible cytochrome P450-2E1 and cytochrome P450 isoforms in exosomes of alcohol-exposed rodents and patients with alcoholism through oxidative and endoplasmic reticulum stress. Hepatol Commun 2017;1:675-690.

17. Theurey P, Rieusset J. Mitochondria-associated membranes response to nutrient availability and role in metabolic diseases. Trends Endocrinol Metab 2017;28:32-45.

18. Wang L, Liu X, Nie J, Zhang J, Kimball SR, Zhang H, et al. ALCAT1 controls mitochondrial etiology of fatty liver diseases, linking defective mitophagy to steatosis. Hepatology 2015;61:486-496.

19. Kawano Y, Cohen DE. Mechanisms of hepatic triglyceride accumulation in non-alcoholic fatty liver disease. J Gastroenterol 2013;48:434-441.

20. Obrowsky S, Chandak PG, Patankar JV, Povoden S, Schlager S, Kershaw $E$, et al. Adipose triglyceride lipase is a TG hydrolase of the small intestine and regulates intestinal PPARa signaling. J Lipid Res 2013;54:425-435.

21. Parker R, Kim SJ, Im GY, Nahas J, Dhesi B, Vergis N, et al. Obesity in acute alcoholic hepatitis increases morbidity and mortality. EBioMedicine 2019;45:511-518.

22. Fernández-Checa JC, Hirano T, Tsukamoto H, Kaplowitz N. Mitochondrial glutathione depletion in alcoholic liver disease. Alcohol 1993;10:469-475.

23. Fabbrini E, Sullivan S, Klein S. Obesity and nonalcoholic fatty liver disease: biochemical, metabolic, and clinical implications. Hepatology 2010;51:679-689.

24. Mandard S, Müller M, Kersten S. Peroxisome proliferator-activated receptor alpha target genes. Cell Mol Life Sci 2004;61:393-416.

25. Shin SY, Kim TH, Wu H, Choi YH, Kim SG. SIRT1 activation by methylene blue, a repurposed drug, leads to AMPK-mediated inhibition of steatosis and steatohepatitis. Eur J Pharmacol 2014;727:115-124.

26. Kim TH, Yang YM, Han CY, Koo JH, Oh H, Kim SS, et al. Ga12 ablation exacerbates liver steatosis and obesity by suppressing USP22/SIRT1-regulated mitochondrial respiration. J Clin Invest 2018;128:5587-5602.

27. Weiskirchen R, Weiskirchen S, Tacke F. Organ and tissue fibrosis: molecular signals, cellular mechanisms and translational implications. Mol Aspects Med 2019;65:2-15.

28. Weiskirchen $R$, Weiskirchen $S$, Tacke F. Recent advances in under- standing liver fibrosis: bridging basic science and individualized treatment concepts. F1000Res 2018;7:F1000 Faculty Rev-921.

29. Lotersztajn S, Julien B, Teixeira-Clerc F, Grenard P, Mallat A. Hepatic fibrosis: molecular mechanisms and drug targets. Annu Rev Pharmacol Toxicol 2005;45:605-628.

30. Gao B, Bataller R. Alcoholic liver disease: pathogenesis and new therapeutic targets. Gastroenterology 2011;141:1572-1585.

31. Marra F, Lotersztajn S. Pathophysiology of NASH: perspectives for a targeted treatment. Curr Pharm Des 2013;19:5250-5269.

32. Kaur J, Debnath J. Autophagy at the crossroads of catabolism and anabolism. Nat Rev Mol Cell Biol 2015;16:461-472.

33. Madrigal-Matute J, Cuervo AM. Regulation of liver metabolism by autophagy. Gastroenterology 2016;150:328-339.

34. Lamming DW, Bar-Peled L. Lysosome: the metabolic signaling hub. Traffic 2019;20:27-38.

35. Boya P, Reggiori F, Codogno P. Emerging regulation and functions of autophagy. Nat Cell Biol 2013;15:713-720.

36. Mizushima N, Levine B, Cuervo AM, Klionsky DJ. Autophagy fights disease through cellular self-digestion. Nature 2008;451:10691075.

37. Schulze RJ, Drižytè K, Casey CA, McNiven MA. Hepatic lipophagy: new insights into autophagic catabolism of lipid droplets in the liver. Hepatol Commun 2017;1:359-369.

38. Singh R, Cuervo AM. Autophagy in the cellular energetic balance. Cell Metab 2011;13:495-504.

39. Deretic V, Saitoh T, Akira S. Autophagy in infection, inflammation and immunity. Nat Rev Immunol 2013;13:722-737.

40. Rubinsztein DC, Codogno P, Levine B. Autophagy modulation as a potential therapeutic target for diverse diseases. Nat Rev Drug Discov 2012;11:709-730.

41. Ding WX, Li M, Chen X, Ni HM, Lin CW, Gao W, et al. Autophagy reduces acute ethanol-induced hepatotoxicity and steatosis in mice. Gastroenterology 2010;139:1740-1752.

42. Donohue TM Jr, Osna NA, Kharbanda KK, Thomes PG. Lysosome and proteasome dysfunction in alcohol-induced liver injury. Liver Research 2019;3:191-205.

43. Li Y, Wang S, Ni HM, Huang H, Ding WX. Autophagy in alcoholinduced multiorgan injury: mechanisms and potential therapeutic targets. Biomed Res Int 2014;2014:498491.

44. Thomes PG, Trambly CS, Fox HS, Tuma DJ, Donohue TM Jr. Acute and chronic ethanol administration differentially modulate hepatic autophagy and transcription factor EB. Alcohol Clin Exp Res 2015;39:2354-2363.

45. Lavallard VJ, Gual P. Autophagy and non-alcoholic fatty liver disease. BioMed Res Int 2014;2014:120179.

46. Shibata M, Yoshimura K, Furuya N, Koike M, Ueno T, Komatsu M, et al. The MAP1-LC3 conjugation system is involved in lipid droplet formation. Biochem Biophys Res Commun 2009;382:419-423. 
47. Yang L, Li P, Fu S, Calay ES, Hotamisligil GS. Defective hepatic autophagy in obesity promotes ER stress and causes insulin resistance. Cell Metab 2010;11:467-478.

48. Tanaka S, Hikita H, Tatsumi T, Sakamori R, Nozaki Y, Sakane S, et al. Rubicon inhibits autophagy and accelerates hepatocyte apoptosis and lipid accumulation in nonalcoholic fatty liver disease in mice. Hepatology 2016;64:1994-2014.

49. Lin CW, Zhang H, Li M, Xiong X, Chen X, Chen X, et al. Pharmacological promotion of autophagy alleviates steatosis and injury in alcoholic and non-alcoholic fatty liver conditions in mice. J Hepatol 2013;58:993-999.

50. Kim YS, Nam HJ, Han CY, Joo MS, Jang K, Jun DW, et al. LXRa activation inhibits autophagy and lipophagy in hepatocytes by dysregulating ATG4B and Rab-8B, reducing mitochondrial fuel oxidation. Hepatology. 2020 Jun 17. doi: 10.1002/hep.31423.

51. Gracia-Sancho J, Guixé-Muntet S. The many-faced role of autophagy in liver diseases. J Hepatol 2018;68:593-594.

52. Tsuchida T, Friedman SL. Mechanisms of hepatic stellate cell activation. Nat Rev Gastroenterol Hepatol 2017;14:397-411.

53. Kim KM, Han CY, Kim JY, Cho SS, Kim YS, Koo JH, et al. Ga12 overexpression induced by miR-16 dysregulation contributes to liver fibrosis by promoting autophagy in hepatic stellate cells. J Hepatol 2018;68:493-504.

54. Tran A, Gual P. Non-alcoholic steatohepatitis in morbidly obese patients. Clin Res Hepatol Gastroenterol 2013;37:17-29.

55. Wang S, Zhu M, Wang Q, Hou Y, Li L, Weng H, et al. Alphafetoprotein inhibits autophagy to promote malignant behaviour in hepatocellular carcinoma cells by activating PI3K/AKT/mTOR signalling. Cell Death Dis 2018;9:1027.

56. Thoen LF, Guimarães EL, Dollé L, Mannaerts I, Najimi M, Sokal E, et al. A role for autophagy during hepatic stellate cell activation. J Hepatol 2011;55:1353-1360.

57. Wannamethee G, Shaper AG. Blood lipids: the relationship with alcohol intake, smoking, and body weight. J Epidemiol Community Health 1992;46:197-202.

58. Zilkens RR, Rich L, Burke V, Beilin LJ, Watts GF, Puddey IB. Effects of alcohol intake on endothelial function in men: a randomized controlled trial. J Hypertens 2003;21:97-103.

59. Volkow ND, Wang GJ, Baler RD. Reward, dopamine and the control of food intake: implications for obesity. Trends Cogn Sci 2011;15:37-46.

60. Seiler $A E$, Henderson $A$, Rubin R. Ethanol inhibits insulin receptor tyrosine kinase. Alcohol Clin Exp Res 2000;24:1869-1872.

61. Lieber CS. New pathway of ethanol metabolism in the liver. Gastroenterology 1970;59:930-937.

62. Lieber CS, DeCarli LM. Hepatotoxicity of ethanol. J Hepatol 1991:12:394-401.

63. Yin H, Hu M, Zhang R, Shen Z, Flatow L, You M. MicroRNA-217 promotes ethanol-induced fat accumulation in hepatocytes by down-regulating SIRT1. J Biol Chem 2012;287:9817-9826.

64. Leung TM, Lu Y, Yan W, Morón-Concepción JA, Ward SC, Ge $X$, et al. Argininosuccinate synthase conditions the response to acute and chronic ethanol-induced liver injury in mice. Hepatology 2012;55:1596-1609.

65. Lehwald N, Tao GZ, Jang KY, Papandreou I, Liu B, Liu B, et al. $\beta$-catenin regulates hepatic mitochondrial function and energy balance in mice. Gastroenterology 2012;143:754-764.

66. Junge J, Horn T, Christoffersen P. Megamitochondria as a diagnostic marker for alcohol induced centrilobular and periportal fibrosis in the liver. Virchows Arch A Pathol Anat Histopathol 1987;410:553-558.

67. Palma E, Ma X, Riva A, lansante $V$, Dhawan A, Wang $S$, et al. Dynamin-1-like protein inhibition drives megamitochondria formation as an adaptive response in alcohol-induced hepatotoxicity. Am J Pathol 2019;189:580-589.

68. Ertunc ME, Hotamisligil GS. Lipid signaling and lipotoxicity in metaflammation: indications for metabolic disease pathogenesis and treatment. J Lipid Res 2016;57:2099-2114.

69. Wang Y, Viscarra J, Kim SJ, Sul HS. Transcriptional regulation of hepatic lipogenesis. Nat Rev Mol Cell Biol 2015;16:678-689.

70. Williams JA, Ni HM, Ding Y, Ding WX. Parkin regulates mitophagy and mitochondrial function to protect against alcohol-induced liver injury and steatosis in mice. Am J Physiol Gastrointest Liver Physiol 2015;309:G324-G340.

71. Singh R, Kaushik S, Wang Y, Xiang Y, Novak I, Komatsu M, et al. Autophagy regulates lipid metabolism. Nature 2009;458:11311135.

72. Ipsen DH, Lykkesfeldt J, Tveden-Nyborg P. Molecular mechanisms of hepatic lipid accumulation in non-alcoholic fatty liver disease. Cell Mol Life Sci 2018;75:3313-3327.

73. Teli MR, Day CP, Burt AD, Bennett MK, James OF. Determinants of progression to cirrhosis or fibrosis in pure alcoholic fatty liver. Lancet 1995;346:987-990.

74. Zhou Z, Wang L, Song Z, Lambert JC, McClain CJ, Kang YJ. A critical involvement of oxidative stress in acute alcohol-induced hepatic TNF-alpha production. Am J Pathol 2003;163:1137-1146.

75. McVicker BL, Rasineni K, Tuma DJ, McNiven MA, Casey CA. Lipid droplet accumulation and impaired fat efflux in polarized hepatic cells: consequences of ethanol metabolism. Int J Hepatol 2012;2012:978136.

76. Crabb DW, Galli A, Fischer M, You M. Molecular mechanisms of alcoholic fatty liver: role of peroxisome proliferator-activated receptor alpha. Alcohol 2004;34:35-38.

77. Bataller R, Rombouts K, Altamirano J, Marra F. Fibrosis in alcoholic and nonalcoholic steatohepatitis. Best Pract Res Clin Gastroenterol 2011;25:231-244 
Yun Seok Kim, et al.

ER stress and autophagy in liver diseases

78. McAlaney J, McMahon J. Establishing rates of binge drinking in the UK: anomalies in the data. Alcohol Alcohol 2006;41:355-357.

79. Mathurin $P$, Deltenre P. Effect of binge drinking on the liver: an alarming public health issue? Gut 2009;58:613-617.

80. Sozio M, Crabb DW. Alcohol and lipid metabolism. Am J Physiol Endocrinol Metab 2008;295:E10-E16.

81. French SW. Ethanol and hepatocellular injury. Clin Lab Med 1996;16:289-306.

82. Savolainen VT, Liesto K, Männikkö A, Penttilä A, Karhunen PJ. Alcohol consumption and alcoholic liver disease: evidence of a threshold level of effects of ethanol. Alcohol Clin Exp Res 1993;17: 1112-1117.

83. Grant BF, Dufour MC, Harford TC. Epidemiology of alcoholic liver disease. Semin Liver Dis 1988;8:12-25.

84. MacSween RN, Burt AD. Histologic spectrum of alcoholic liver disease. Semin Liver Dis 1986;6:221-232.

85. Zimatkin SM, Pronko SP, Vasiliou V, Gonzalez FJ, Deitrich RA. Enzymatic mechanisms of ethanol oxidation in the brain. Alcohol Clin Exp Res 2006;30:1500-1505.

86. Osna NA, Donohue TM Jr, Kharbanda KK. Alcoholic liver disease: pathogenesis and current management. Alcohol Res 2017;38:147161.

87. Milne GL, Sanchez SC, Musiek ES, Morrow JD. Quantification of F2-isoprostanes as a biomarker of oxidative stress. Nat Protoc 2007:2:221-226.

88. Morrow JD, Hill KE, Burk RF, Nammour TM, Badr KF, Roberts LJ 2nd. A series of prostaglandin F2-like compounds are produced in vivo in humans by a non-cyclooxygenase, free radical-catalyzed mechanism. Proc Natl Acad Sci U S A 1990;87:9383-9387.

89. Iuliano L, Praticò D, Greco C, Mangieri E, Scibilia G, FitzGerald GA, et al. Angioplasty increases coronary sinus F2-isoprostane formation: evidence for in vivo oxidative stress during PTCA. J Am Coll Cardiol 2001;37:76-80.

90. Murphy MP. How mitochondria produce reactive oxygen species. Biochem J 2009;417:1-13.

91. Heo MJ, Kim TH, You JS, Blaya D, Sancho-Bru P, Kim SG. Alcohol dysregulates miR-148a in hepatocytes through Fox01, facilitating pyroptosis via TXNIP overexpression. Gut 2019;68:708-720.

92. Chen Y, Singh S, Matsumoto A, Manna SK, Abdelmegeed MA, Golla $S$, et al. Chronic glutathione depletion confers protection against alcohol-induced steatosis: implication for redox activation of AMP-activated protein kinase pathway. Sci Rep 2016;6:29743.

93. Rinella ME. Nonalcoholic fatty liver disease: a systematic review. JAMA 2015;313:2263-2273.

94. Targher G. Non-alcoholic fatty liver disease as driving force in coronary heart disease? Gut 2017;66:213-214.

95. Lassailly G, Caiazzo R, Pattou F, Mathurin P. Perspectives on treatment for nonalcoholic steatohepatitis. Gastroenterology
2016;150:1835-1848.

96. European Association for the Study of the Liver (EASL); European Association for the Study of Diabetes (EASD); European Association for the Study of Obesity (EASO). EASL-EASD-EASO clinical practice Guidelines for the management of non-alcoholic fatty liver disease. J Hepatol 2016;64:1388-1402.

97. Ma K, Saha PK, Chan L, Moore DD. Farnesoid X receptor is essential for normal glucose homeostasis. J Clin Invest 2006;116:11021109.

98. Ploton M, Mazuy C, Gheeraert C, Dubois V, Berthier A, DuboisChevalier J, et al. The nuclear bile acid receptor FXR is a PKA- and FOXA2-sensitive activator of fasting hepatic gluconeogenesis. J Hepatol 2018;69:1099-1109.

99. Brunt EM, Kleiner DE. Challenges in the hepatic histopathology in non-alcoholic fatty liver disease. Gut 2017;66:1539-1540.

100. Arab JP, Sehrawat TS, Simonetto DA, Verma VK, Feng D, Tang T, et al. An open-label, dose-escalation study to assess the safety and efficacy of IL-22 agonist F-652 in patients with alcohol-associated hepatitis. Hepatology 2020;72:441-453.

101. Spalinger MR, Atrott K, Baebler K, Schwarzfischer M, Melhem H, Peres DR, et al. Administration of the hyper-immune bovine colostrum extract IMM-124E ameliorates experimental murine colitis. J Crohns Colitis 2019;13:785-797.

102. Mantegazza C, Molinari P, D'Auria E, Sonnino M, Morelli L, Zuccotti GV. Probiotics and antibiotic-associated diarrhea in children: a review and new evidence on Lactobacillus rhamnosus GG during and after antibiotic treatment. Pharmacol Res 2018;128:63-72.

103. Petrasek J, Bala S, Csak T, Lippai D, Kodys K, Menashy V, et al. IL-1 receptor antagonist ameliorates inflammasome-dependent alcoholic steatohepatitis in mice. J Clin Invest 2012;122:3476-3489.

104. Iracheta-Vellve A, Calenda CD, Petrasek J, Ambade A, Kodys K, Adorini $L$, et al. FXR and TGR5 agonists ameliorate liver injury, steatosis, and inflammation after binge or prolonged alcohol feeding in mice. Hepatol Commun 2018;2:1379-1391.

105. Hakami AY, Alshehri FS, Althobaiti YS, Sari Y. Effects of orally administered augmentin on glutamate transporter 1, cystineglutamate exchanger expression and ethanol intake in alcoholpreferring rats. Behav Brain Res 2017;320:316-322.

106. Di Miceli M, Gronier B. Pharmacology, systematic review and recent clinical trials of metadoxine. Rev Recent Clin Trials 2018;13:114125

107. Nikou T, loannidis A, Zoga M, Tzavellas E, Paparrigopoulos T, Magana $\mathrm{M}$, et al. Alteration in the concentrations of interleukin-7 (IL-7), interleukin-10 (IL-10) and granulocyte colony stimulating factor (G-CSF) in alcohol-dependent individuals without liver disease, during detoxification therapy. Drug Alcohol Depend 2016;163:7783.

108. Choi WM, Kim HH, Kim MH, Cinar R, Yi HS, Eun HS, et al. Gluta- 
mate signaling in hepatic stellate cells drives alcoholic steatosis. Cell Metab 2019;30:877-889.e7.

109. Ge X, Antoine DJ, Lu Y, Arriazu E, Leung TM, Klepper AL, et al. High mobility group box-1 (HMGB1) participates in the pathogenesis of alcoholic liver disease (ALD). J Biol Chem 2014;289:2267222691.

110. Seo W, Gao Y, He Y, Sun J, Xu H, Feng D, et al. ALDH2 deficiency promotes alcohol-associated liver cancer by activating oncogenic pathways via oxidized DNA-enriched extracellular vesicles. J Hepatol 2019;71:1000-1011.

111. Lee JH, Shim YR, Seo W, Kim MH, Choi WM, Kim HH, et al. Mitochondrial double-stranded RNA in exosome promotes interleukin-17 production through toll-like receptor 3 in alcohol-associated liver injury. Hepatology 2020;72:609-625.

112. McCullough RL, McMullen MR, Poulsen KL, Kim A, Medof ME, Nagy LE. Anaphylatoxin receptors C $3 a R$ and $C 5 a R 1$ are important factors that influence the impact of ethanol on the adipose secretome. Front Immunol 2018;9:2133.

113. Cho YE, Kim DK, Seo W, Gao B, Yoo SH, Song BJ. Fructose promotes leaky gut, endotoxemia, and liver fibrosis through ethanolinducible cytochrome P450-2E1-mediated oxidative and nitrative stress. Hepatology. 2019 Apr 8. doi: 10.1002/hep.30652.

114. Shao T, Zhao C, Li F, Gu Z, Liu L, Zhang L, et al. Intestinal HIF-1a deletion exacerbates alcoholic liver disease by inducing intestinal dysbiosis and barrier dysfunction. J Hepatol 2018;69:886-895.

115. Forsyth CB, Farhadi A, Jakate SM, Tang Y, Shaikh M, Keshavarzian A. Lactobacillus GG treatment ameliorates alcohol-induced intestinal oxidative stress, gut leakiness, and liver injury in a rat model of alcoholic steatohepatitis. Alcohol 2009;43:163-172.

116. Hartmann $P$, Hochrath $K$, Horvath $A$, Chen $P$, Seebauer $C T$, Llorente $C$, et al. Modulation of the intestinal bile acid/farnesoid $X$ receptor/ fibroblast growth factor 15 axis improves alcoholic liver disease in mice. Hepatology 2018;67:2150-2166.

117. Brandl K, Hartmann P, Jih LJ, Pizzo DP, Argemi J, Ventura-Cots $M$, et al. Dysregulation of serum bile acids and FGF19 in alcoholic hepatitis. J Hepatol 2018;69:396-405.

118. Wang L, Fouts DE, Stärkel P, Hartmann P, Chen P, Llorente C, et al. Intestinal REG3 lectins protect against alcoholic steatohepatitis by reducing mucosa-associated microbiota and preventing bacterial translocation. Cell Host Microbe 2016;19:227-239.

119. Zhou J, Waskowicz LR, Lim A, Liao XH, Lian B, Masamune $H$, et al. A liver-specific thyromimetic, VK2809, decreases hepatosteatosis in glycogen storage disease type la. Thyroid 2019;29:1158-1167.

120. Boubia B, Poupardin O, Barth M, Binet J, Peralba P, Mounier L, et al. Design, synthesis, and evaluation of a novel series of indole sulfonamide peroxisome proliferator activated receptor (PPAR) $a / \gamma / \delta$ triple activators: discovery of lanifibranor, a new antifibrotic clinical candidate. J Med Chem 2018;61:2246-2265.
121. Tully DC, Rucker PV, Chianelli D, Williams J, Vidal A, Alper PB, et al. Discovery of tropifexor (LJN452), a highly potent non-bile acid FXR agonist for the treatment of cholestatic liver diseases and nonalcoholic steatohepatitis (NASH). J Med Chem 2017;60:9960-9973.

122. He YL, Haynes $W$, Meyers CD, Amer A, Zhang Y, Mahling $P$, et al. The effects of licogliflozin, a dual SGLT1/2 inhibitor, on body weight in obese patients with or without diabetes. Diabetes Obes Metab 2019;21:1311-1321.

123. Li S, Ghoshal S, Sojoodi M, Arora G, Masia R, Erstad DJ, et al. The farnesoid $X$ receptor agonist EDP-305 reduces interstitial renal fibrosis in a mouse model of unilateral ureteral obstruction. FASEB J 2019;33:7103-7112.

124. Newsome PN, Palmer M, Freilich B, Sheikh MY, Sheikh A, Sarles $\mathrm{H}$, et al. Volixibat in adults with non-alcoholic steatohepatitis: 24week interim analysis from a randomized, phase II study. J Hepatol 2020;73:231-240.

125. Sanyal A, Charles ED, Neuschwander-Tetri BA, Loomba R, Harrison SA, Abdelmalek MF, et al. Pegbelfermin (BMS-986036), a PEGylated fibroblast growth factor 21 analogue, in patients with nonalcoholic steatohepatitis: a randomised, double-blind, placebocontrolled, phase 2a trial. Lancet 2019;392:2705-2717.

126. Barreyro FJ, Holod S, Finocchietto PV, Camino AM, Aquino JB, Avagnina $A$, et al. The pan-caspase inhibitor emricasan (IDN6556) decreases liver injury and fibrosis in a murine model of nonalcoholic steatohepatitis. Liver Int 2015;35:953-966.

127. Harrison SA, Marri SR, Chalasani N, Kohli R, Aronstein W, Thompson GA, et al. Randomised clinical study: GR-MD-02, a galectin-3 inhibitor, vs. placebo in patients having non-alcoholic steatohepatitis with advanced fibrosis. Aliment Pharmacol Ther 2016;44:11831198.

128. Tacke F. Cenicriviroc for the treatment of non-alcoholic steatohepatitis and liver fibrosis. Expert Opin Investig Drugs 2018;27:301-311.

129. Iruarrizaga-Lejarreta $M$, Varela-Rey M, Fernández-Ramos $D$, Martínez-Arranz I, Delgado TC, Simon J, et al. Role of Aramchol in steatohepatitis and fibrosis in mice. Hepatol Commun 2017;1:911927.

130. Harrison SA, Bashir MR, Guy CD, Zhou R, Moylan CA, Frias JP, et al. Resmetirom (MGL-3196) for the treatment of non-alcoholic steatohepatitis: a multicentre, randomised, double-blind, placebocontrolled, phase 2 trial. Lancet 2019;394:2012-2024.

131. Ratziu V, Harrison SA, Francque S, Bedossa P, Lehert P, Serfaty L, et al. Elafibranor, an agonist of the peroxisome proliferator-activated receptor- $a$ and $-\delta$, induces resolution of nonalcoholic steatohepatitis without fibrosis worsening. Gastroenterology 2016;150:11471159.e5.

132. Younossi ZM, Ratziu V, Loomba R, Rinella M, Anstee QM, Goodman $Z$, et al. Obeticholic acid for the treatment of non-alcoholic steatohepatitis: interim analysis from a multicentre, randomised, 
Yun Seok Kim, et al.

ER stress and autophagy in liver diseases

placebo-controlled phase 3 trial. Lancet 2019;394:2184-2196.

133. Schuster S, Feldstein AE. NASH: novel therapeutic strategies targeting ASK1 in NASH. Nat Rev Gastroenterol Hepatol 2017;14:329330.

134. Akuta N, Watanabe C, Kawamura Y, Arase Y, Saitoh S, Fujiyama $\mathrm{S}$, et al. Effects of a sodium-glucose cotransporter 2 inhibitor in nonalcoholic fatty liver disease complicated by diabetes mellitus: preliminary prospective study based on serial liver biopsies. Hepatol Commun 2017;1:46-52.

135. Grohmann M, Wiede F, Dodd GT, Gurzov EN, Ooi GJ, Butt T, et al. Obesity drives STAT-1-dependent NASH and STAT-3-dependent HCC. Cell 2018;175:1289-1306.e20.

136. Andres-Hernando A, Orlicky DJ, Kuwabara M, Ishimoto T, Nakagawa $T$, Johnson RJ, et al. Deletion of fructokinase in the liver or in the intestine reveals differential effects on sugar-induced metabolic dysfunction. Cell Metab 2020;32:117-127.e3.

137. Kim TH, Koo JH, Heo MJ, Han CY, Kim YI, Park SY, et al. Overproduction of inter-a-trypsin inhibitor heavy chain 1 after loss of Ga13 in liver exacerbates systemic insulin resistance in mice. Sci Transl
Med 2019;11:eaan4735.

138. Wang X, Cai B, Yang X, Sonubi 00, Zheng Z, Ramakrishnan R, et al. Cholesterol stabilizes TAZ in hepatocytes to promote experimental non-alcoholic steatohepatitis. Cell Metab 2020;31:969986.e7.

139. Liu D, Zhang P, Zhou J, Liao R, Che Y, Gao MM, et al. TNFAIP3 interacting protein 3 overexpression suppresses nonalcoholic steatohepatitis by blocking TAK1 activation. Cell Metab 2020;31:726740.e8.

140. Simon J, Nuñez-García M, Fernández-Tussy P, Barbier-Torres $L$, Fernández-Ramos D, Gómez-Santos B, et al. Targeting hepatic glutaminase 1 ameliorates non-alcoholic steatohepatitis by restoring very-low-density lipoprotein triglyceride assembly. Cell Metab 2020;31:605-622.e10.

141. Verboom L, Martens A, Priem D, Hoste E, Sze M, Vikkula H, et al. OTULIN prevents liver inflammation and hepatocellular carcinoma by inhibiting FADD- and RIPK1 kinase-mediated hepatocyte apoptosis. Cell Rep 2020;30:2237-2247.e6. 